\title{
Acute Changes in Pacing Threshold and R- or P-Wave Amplitude During Permanent Pacemaker Implantation
}

\author{
Michael de Buitleir, MD, William H. Kou, MD, Stephen Schmaltz, MPH, and Fred Morady, MD
}

This study examines the changes in pacing threshold and R- or P-wave amplitude during the first 30 minutes after implantation of tined and screw-in leads. The leads examined were those of 1 manufacturer (Medtronic) and consisted of 3 ventricular pacing leads (model numbers 6957 unipolar screwin [11 patients], 6961 unipolar tined [12 patients] and 6962 bipolar tined [7 patients]) and 1 atrial lead (model number 6957 J unipolar screw-in [10 patients]). After optimal lead position was obtained fluoroscopically in the right ventricular apex or right atrium, the pacing threshold and $R$ - or $P$ wave amplitudes were measured at 5-minute intervals for $\mathbf{3 0}$ minutes.

The acute ventricular pacing threshold with the screw-in lead was significantly higher than with the tined lead $(0.84 \pm 0.17$ vs $0.58 \pm 0.15$ volts; $p$ $<0.001)$. There was a significant $(p<0.001)$ acute decrease in the ventricular pacing threshold with both lead types, with the maximum decrease occurring 5 minutes after lead implantation. There was a significant acute increase in $\mathbf{R}$-wave size with the ventricular screw-in lead that peaked $\mathbf{2 0}$ minutes after lead Implantation (11.9 \pm 3.0 to $14.7 \pm 4.1$ $\mathrm{mV}$; $\mathrm{p}<0.001$ ). The atrial screw-in lead behaved in a manner identical to its counterpart in the ventricle. In conclusion, there are acule changes in the pacing threshold and R- or P-wave amplitude obtained with tined and screw-in pacing leads. In some patients, a pacing threshold or R- or P-wave amplitude that is initially unacceptable may improve to an acceptable level over 15 to 20 minutes without further lead manipulation, especially when an atrial screw-in lead is used.

(Am J Cardiol 1990,65,999-1003)

From the Department of Internal Medicine, Division of Cardiology, and the Clinical Research Center, University of Michigan Medical Center, Ann Arbor, Michigan. Manuscript received September 25, 1989; revised manuscript received and accepted December 18, 1989.

Address for reprints: Michael de Buitleir, MD, Division of Cardiology, Department of Internal Medicine, University of Michigan Medical Center, UH B1/F245, 1500 East Medical Center Drive, Ann Arbor, Michigan 48109-0022.
I he acute pacing threshold and R- or P-wave amplitudes are important in identifying an adequate lead position during permanent pacemaker implantation. A number of factors influence these parameters including the type of lead used, the lead position within the cardiac chamber and the extent of contact between the lead tip and viable myocardium. Because of contrasting means of myocardial contact, differences in acute pacing parameters might be expected with tined and screw-in lead tip configurations. However, no studies to date have performed a systematic analysis of the acute pacing and sensing parameters with these leads. The purpose of this study was to compare the immediate pacing thresholds and sensing characteristics of tined and screw-in leads and to examine acute changes in these parameters during the first 30 minutes after implantation.

\section{METHODS}

Subjects: The study protocol was performed during implantation of a clinically indicated permanent pacemaker for which all patients had given informed consent. Thirty-five patients were included in the study. Their mean age was $66 \pm 15$ years (range 28 to 90 ) and 20 were men. The indication for permanent pacemaker implantation was sick sinus syndrome in 15 patients, carotid sinus syndrome in 6 patients and atrioventricular block in 14 patients.

Pacing leads: Four Medtronic endocardial pacing leads were compared. The leads of 1 manufacturer were used so as to maintain uniformity for comparison. Eleven patients had a unipolar ventricular screw-in lead (model 6957). The electrode on this lead is a platinum alloy helix that provides active fixation to the endocardium. The helix is advanced or retracted by rotating the connector pin with a fixation tool. The lead has a nickel alloy conductor and polyurethane insulation. Twelve patients had a unipolar ventricular tined lead (model 6961). This lead has a platinum alloy ring-shaped tip electrode. In addition, there are 4 silicone rubber tines near the electrode tip, a nickel alloy conductor and silicone rubber insulation. Seven patients had a bipolar ventricular tined lead (model 6962); this lead is identical to the unipolar ventricular tined lead (model 6961), except that there is a second ring electrode near the tip. Ten patients had a unipolar atrial screw-in lead (model $6957 \mathrm{~J}$ ). This lead is identical to its counterpart in the ventricle (model 6957) except that the distal segment is J-shaped. This modification is designed to facilitate electrode placement in the right atrial appendage, on the lateral right atrial wall or on the atrial septum. 


\begin{tabular}{|c|c|c|c|c|c|c|}
\hline \multirow[b]{3}{*}{ Minutes } & \multicolumn{6}{|l|}{ Threshold } \\
\hline & \multicolumn{2}{|l|}{6957} & \multicolumn{2}{|l|}{6961} & \multicolumn{2}{|l|}{6962} \\
\hline & Volts & $\mathrm{mA}$ & Volts & $\mathrm{mA}$ & Volts & $\mathrm{mA}$ \\
\hline 0 & $0.84 \pm 0.17$ & $1.24 \pm 0.40$ & $0.58 \pm 0.15$ & $0.91 \pm 0.25$ & $0.64 \pm 0.26$ & $0.91 \pm 0.36$ \\
\hline 5 & $0.69 \pm 0.14^{\ddagger}$ & $1.04 \pm 0.32^{\ddagger}$ & $0.53 \pm 0.12^{\ddagger}$ & $0.84 \pm 0.16^{\ddagger}$ & $0.51 \pm 0.14^{\ddagger}$ & $0.76 \pm 0.16^{\ddagger}$ \\
\hline 10 & $0.66 \pm 0.12^{\ddagger}$ & $1.00 \pm 0.22^{\ddagger}$ & $0.51 \pm 0.12^{\ddagger}$ & $0.81 \pm 0.18^{\ddagger}$ & $0.51 \pm 0.10^{\ddagger}$ & $0.73 \pm 0.09^{\ddagger}$ \\
\hline 15 & $0.65 \pm 0.10^{\ddagger}$ & $1.00 \pm 0.27^{\ddagger}$ & $0.51 \pm 0.13^{\ddagger}$ & $0.77 \pm 0.17^{\ddagger}$ & $0.53 \pm 0.07^{\ddagger}$ & $0.76 \pm 0.16^{\ddagger}$ \\
\hline 20 & $0.65 \pm 0.09^{\ddagger}$ & $1.00 \pm 0.20^{\ddagger}$ & $0.48 \pm 0.10^{\ddagger}$ & $0.76 \pm 0.19^{\ddagger}$ & $0.51 \pm 0.07^{\ddagger}$ & $0.76 \pm 0.10^{\ddagger}$ \\
\hline 25 & $0.67 \pm 0.10^{\ddagger}$ & $1.00 \pm 0.23^{\dagger}$ & $0.47 \pm 0.10^{\dagger}$ & $0.75 \pm 0.17^{\dagger}$ & $0.51 \pm 0.07^{\dagger}$ & $0.73 \pm 0.17^{\dagger}$ \\
\hline \multirow[t]{3}{*}{30} & $0.65 \pm 0.09^{\ddagger}$ & $1.00 \pm 0.20^{\ddagger}$ & $0.47 \pm 0.10^{\ddagger}$ & $0.73 \pm 0.20 \neq$ & $0.53 \pm 0.07^{\ddagger}$ & $0.77 \pm 0.09^{\ddagger}$ \\
\hline & \multicolumn{3}{|c|}{ Resistance (ohms) } & \multicolumn{3}{|l|}{ R Wave (mV) } \\
\hline & 6957 & 6961 & 6962 & 6957 & 6961 & 6962 \\
\hline 0 & $658 \pm 207$ & $581 \pm 78$ & $618 \pm 93$ & $12 \pm 3$ & $13 \pm 9$ & $17 \pm 8$ \\
\hline 5 & $625 \pm 161$ & $584 \pm 66$ & $585 \pm 83$ & $12 \pm 4$ & $13 \pm 9$ & $17 \pm 7$ \\
\hline 10 & $632 \pm 156$ & $567 \pm 70$ & $625 \pm 103$ & $13 \pm 4$ & $13 \pm 9$ & $17 \pm 8$ \\
\hline 15 & $637 \pm 167$ & $602 \pm 67$ & $600 \pm 82$ & $14 \pm 3^{\dagger}$ & $14 \pm 9$ & $18 \pm 7$ \\
\hline 20 & $627 \pm 150$ & $620 \pm 107$ & $586 \pm 84$ & $15 \pm 4^{\ddagger}$ & $14 \pm 9$ & $17 \pm 7$ \\
\hline 25 & $648 \pm 176$ & $621 \pm 88$ & $593 \pm 69$ & $15 \pm 4^{\ddagger}$ & $14 \pm 9^{*}$ & $18 \pm 7$ \\
\hline 30 & $630 \pm 191$ & $632 \pm 105$ & $585 \pm 76$ & $15 \pm 5^{\ddagger}$ & $14 \pm 9^{\dagger}$ & $17 \pm 8$ \\
\hline
\end{tabular}

Pacing lead implantation and threshold determination: Venous access was obtained through a subclavian or cephalic vein. The ventricular lead was advanced under fluoroscopic guidance and positioned at the right ventricular apex. The atrial lead was positioned in a similar manner either in the right atrial appendage (7 patients) or on the atrial septum (3 patients). After a stable lead position was achieved, serial measurements of pacing threshold, resistance and filtered R- or P-wave amplitude were obtained at 5-minute intervals for 30 minutes. These data were measured with a pacing system analyzer (Medtronic Inc., model 5311). During Rand $\mathrm{P}$-wave measurement the largest amplitude signal detected during a 2-second sampling period was recorded. Unipolar measurements were made with the indif- ferent electrode connected to the tissues of the pacemaker pocket.

The pacing threshold was measured by pacing at a rate of 80 to $100 \mathrm{pulses} / \mathrm{min}$ with stimuli that had a pulse width of $0.5 \mathrm{~ms}$ and an initial amplitude of 1.5 volts. The amplitude was decreased in steps of 0.1 volt, and the pacing threshold was defined as the lowest pulse amplitude at which $100 \%$ ventricular or atrial capture was maintained for at least 15 seconds during normal respiration. The current, resistance and R- or P-wave amplitudes then were measured with the pacing system analyzer. If the initial threshold in the atrium or ventricle was $>1.3$ volts, the lead was repositioned regardless of the fluoroscopic position. However, if the initial threshold remained $>1.3$ volts after $\geq 2$ attempts at re-
FIQURE 1. Acute pacing threshold data over the first 30 minutes after lead implantation in all patients in whom the baseline threshold was suboptimal ( $\geq 1$ volt).

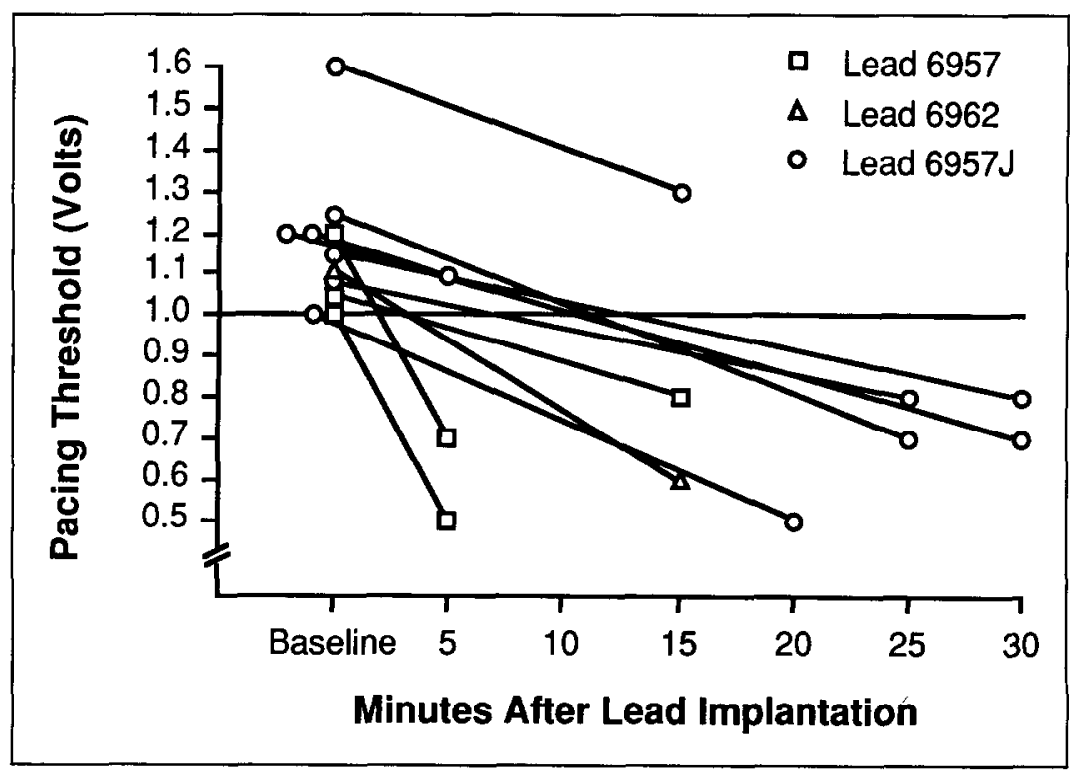


positioning, the higher baseline threshold was accepted. A decision to reposition the lead was based solely on the capture threshold criterion.

Statistical analysis: The data for the 3 ventricular pacing leads and the single atrial pacing lead were analyzed separately. A repeated measures analysis of variance was used to test for differences between the 3 ventricular pacing leads, for changes over time and for interactions between lead and time point differences.' Within each group, different time points were compared using Fisher's least significant difference multiple comparisons procedure. A p value $<0.05$ was considered significant and all data are expressed as mean \pm 1 standard deviation.

\section{RESULTS}

Pacing thresholds: The mean pacing thresholds for both the unipolar and bipolar tined leads were significantly lower than that for the unipolar screw-in lead ( $p$ $<0.001$ ) (Table I). The pacing thresholds for the unipolar and bipolar tined leads were not significantly different from each other. Comparison of the screw-in lead in the atrium and the ventricle revealed that when individual time points were compared, there was no significant difference in pacing threshold. However, if all the time points were combined, the average pacing threshold in the atrium ( 0.88 volt) was significantly higher than that in the ventricle $(0.69$ volt; $p<0.01)$.

The thresholds for all 3 ventricular leads at baseline were significantly higher than at 5 minutes and all subsequent time points $(\mathrm{p}<0.001)$. The threshold at $5 \mathrm{~min}-$ utes was not significantly different from the threshold at any of the later times. The majority of patients displayed the group trend. The improvement was greater in some patients than in others and in no patient did values deteriorate significantly during the period of observation. There were no significant interactions between the thresholds of any of the 3 leads and time. This indicates that the 3 leads displayed the same downward trend in acute pacing threshold over the first 30 minutes after implantation. For the atrial screw-in lead (Table II), the pacing threshold at baseline was also significantly higher than at 5 minutes and all subsequent time points $(p<0.001)$. The threshold at 5 minutes was not significantly different from the thresholds at any of the later times.

Figure 1 shows the acute pacing threshold data in all patients in whom the threshold at baseline was suboptimal ( $\geq 1$ volt). There were 3 patients with a unipolar ventricular screw-in lead, 1 with a bipolar ventricular tined lead and 7 with a unipolar atrial screw-in lead. In all patients except for 2 who had the atrial screw-in lead, the threshold fell to $<1$ volt within 30 minutes of lead implantation. In some patients the maximum improvement occurred by 5 minutes, in others by $15 \mathrm{~min}-$ utes and in others by 30 minutes.

Resistances There were no significant differences in the measured resistance between any of the 3 ventricular pacing leads examined (Table I). There were no significant changes in the measured resistance over time for either the atrial or ventricular leads (Table I and II).
TABLE II Atrial Pacing Threshold, Current, Resistance and P-Wave at Baseline and at 5-minute Intervals for 30 Minutes for the 6957J (Medtronic) Atrial Pacing Lead

\begin{tabular}{|cllll|}
\hline Minutes & $\begin{array}{l}\text { Threshold } \\
\text { (volts) }\end{array}$ & $\begin{array}{l}\text { Current } \\
(\mathrm{mA})\end{array}$ & $\begin{array}{l}\text { Resistance } \\
\text { (ohms) }\end{array}$ & $\begin{array}{l}\text { P wave } \\
(\mathrm{mV})\end{array}$ \\
\hline 0 & $1.07 \pm 0.27$ & $1.95 \pm 0.73$ & $545 \pm 139$ & $3.7 \pm 2.2$ \\
5 & $0.92 \pm 0.31^{\dagger}$ & $1.70 \pm 0.71^{\dagger}$ & $532 \pm 147$ & $4.0 \pm 2.5$ \\
10 & $0.93 \pm 0.31^{\ddagger}$ & $1.75 \pm 0.80^{*}$ & $524 \pm 97$ & $4.5 \pm 2.7^{*}$ \\
15 & $0.86 \pm 0.28^{\ddagger}$ & $1.59 \pm 0.67^{\ddagger}$ & $540 \pm 162$ & $4.7 \pm 3.0^{\dagger}$ \\
20 & $0.82 \pm 0.28^{\ddagger}$ & $1.50 \pm 0.70^{\ddagger}$ & $522 \pm 117$ & $4.8 \pm 2.8^{\ddagger}$ \\
25 & $0.79 \pm 0.28^{\ddagger}$ & $1.46 \pm 0.70^{\ddagger}$ & $518 \pm 104$ & $5.2 \pm 3.3^{*}$ \\
30 & $0.77 \pm 0.28^{\ddagger}$ & $1.42 \pm 0.71^{\ddagger}$ & $539 \pm 126$ & $5.2 \pm 3.1^{\ddagger}$ \\
\hline \multicolumn{5}{|c}{ significant. } \\
\hline \multicolumn{5}{|l}{} \\
\hline
\end{tabular}

R- and P-wave amplitudes: There were no significant differences in the $\mathrm{R}$-wave amplitude between the 3 ventricular pacing leads $(\mathrm{p}=0.49)$ (Table $\mathrm{I})$. However, there was a significant increase over time in the $\mathrm{R}$-wave amplitude for the 2 unipolar leads ( $p<0.006$ ). For the unipolar screw-in lead, the $R$ wave at 15 minutes was significantly larger than the baseline $(p<0.01)$ and the $R$ wave at 20,25 and 30 minutes displayed a further significant increment. In the case of the unipolar tined lead, the $R$ wave at 25 minutes was significantly larger than the baseline $(p<0.05)$ and the $R$ wave at 30 minutes displayed a further small increment $(\mathrm{p}<0.01)$. For the bipolar tined lead, the size of the $R$ wave at baseline was not significantly different than any of the subsequent time points.

There was also a significant increase in the magnitude of the measured $\mathrm{P}$ wave over time (Table II). Compared to baseline, the $P$ wave was significantly larger at $10(p<0.05)$ and 15 minutes $(p<0.01)$. There was a further significant increment in the size of the $P$ wave at 20 minutes $(p<0.001)$ and this was maintained at the subsequent time points.

Figure 2 shows the acute R-wave amplitude in all patients in whom the baseline $R$ wave was suboptimal $(<7 \mathrm{mV})$. There were 4 such patients, 2 with a unipolar tined lead and 2 with a bipolar tined lead. In 3 of the 4 patients, the $R$ wave at 30 minutes was larger than at baseline. In 1 patient the $R$ wave improved and then disimproved but this patient was the exception (Figure 2). Figure 3 shows the acute $P$-wave amplitude in all patients in whom the baseline $P$ wave was $<5 \mathrm{mV}$. In the majority of these patients, the $P$ wave at 30 minutes was larger than at baseline.

\section{DISCUSSION}

Comparison of different leads: The results of this study demonstrate that the acute ventricular pacing threshold obtained with a screw-in lead is significantly higher than that obtained with a representative tined lead. There is no difference in the pacing threshold between a unipolar and a bipolar tined lead. R-wave size does not differ significantly between the screw-in and tined lead configurations.

Changes over time: There is a significant decrease in the acute pacing threshold with both the screw-in and tined leads, with the maximum change generally occur- 
ring by 5 minutes after lead implantation. The practical importance of this finding arises when a lead has a satisfactory radiographic position but a marginal acute pacing threshold. In this situation it is reasonable to wait 5 minutes and reassess the pacing threshold. Our data suggest that the pacing threshold often may improve to an acceptable level, obviating the need for further lead manipulation.

With the ventricular screw-in lead, there is a highly significant acute increase in R-wave size, which peaks 20 minutes after lead implantation. Therefore, in a patient with a marginal $\mathrm{R}$ wave $(<5 \mathrm{mV}$ ) immediately after lead implantation in whom other pacing parameters are satisfactory, it is reasonable to accept this $\mathbf{R}$ wave in anticipation of improvement within 15 or 20 minutes. With the unipolar ventricular tined lead, there is a similar but less prominent acute increase in $\mathrm{R}$-wave size at 25 minutes after lead implantation. However, there is no significant acute increase in $\mathrm{R}$-wave size with the bipolar ventricular tined lead.

Our study demonstrates that the screw-in lead behaves in an identical fashion in the atrium as in the ventricle. There is a highly significant decrease in the atrial pacing threshold over the first 5 minutes after lead implantation and also a highly significant increase in P-wave size over the first 20 minutes after lead implantation. Thus, as in the case of the ventricular lead, if the atrial lead is in a stable position radiographically with marginal thresholds, it is reasonable to remeasure these thresholds after a short time in anticipation of a significant improvement. As demonstrated in Figure 3, most patients with a marginal baseline P-wave amplitude will show significant improvement within $30 \mathrm{~min}$ utes to clinically acceptable values.
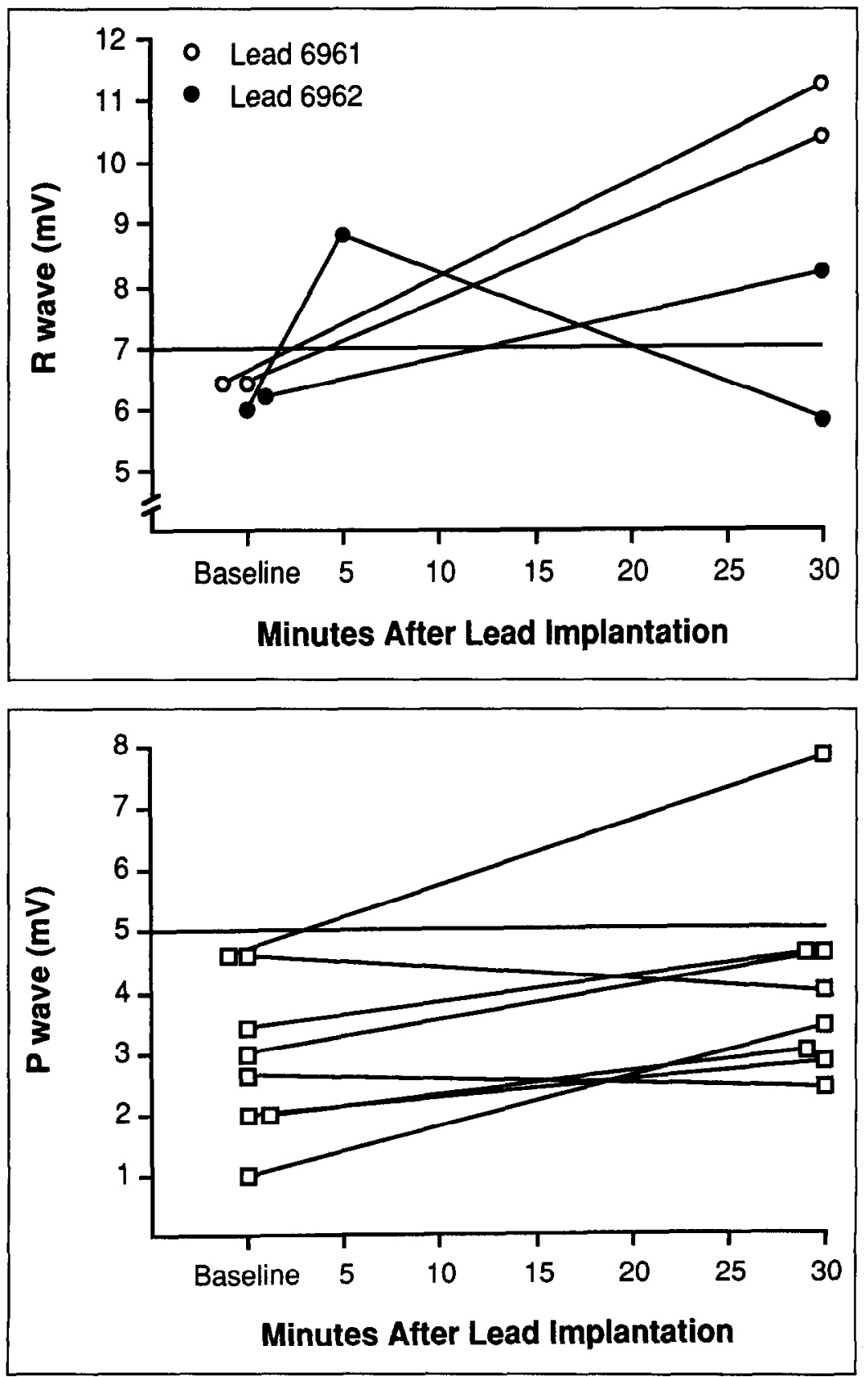

FGURE 2. R-wave amplitude over the first $\mathbf{3 0}$ minutes after lead implantation in all patients in whom the baseline $R$ wave was suboptimal $(<\mathbf{7 ~ m V}$ ).
FIGURE 3. P-wave amplitude (lead 6957J) over the first 30 minutes after lead implantation in all patients in whom the baseline $P$ wave was $<5 \mathrm{mV}$. 
Possible mechanisms: Implantation of the screw-in lead causes localized endocardial injury. This injury possibly may acutely raise the pacing threshold and impair the sensing function of the screw-in lead. With the passage of time after lead implantation this area of injury may recede rapidly, with reabsorption of edema fluid and improvement in pacing and sensing parameters. In contrast, the tined lead would not be expected to injure the endocardium. Thus it would seem predictable that the initial pacing threshold is lower with this lead than with the screw-in lead. Improvement in the acute pacing and sensing characteristics with the tined lead may be due to the gradual improvement in contact between the lead tip and the endocardium over the first 30 minutes after lead implantation.

Prior studies: To our knowledge, no prior studies have examined the evolution of pacing and sensing parameters over the first 30 minutes after implantation of permanent pacemaker leads in patients. However, a number of studies have examined these data acutely and over a variable follow-up period. Mond and Sloman $^{2}$ implanted a unipolar ventricular tined lead (Medtronic 6961) in 100 patients and achieved an average acute pacing threshold of 0.59 volt, current of $0.92 \mathrm{~mA}$, resistance of $560 \mathrm{ohms}$ and $\mathrm{R}$-wave of $8 \mathrm{mV}$. These data are very similar to those in our study. Berman et $\mathrm{al}^{3}$ and Scoblionko and Rolett ${ }^{4}$ examined changes in the pacing threshold at frequent intervals over 1 year and 12 weeks, respectively, by means of pulse-width programming. However, their data are not comparable to ours. Holmes et $\mathrm{al}^{5}$ implanted a bipolar ventricular tined lead (Medtronic 6962) in 50 patients and found an acute mean stimulation threshold of $0.43 \pm 0.14$ volt, current of $0.78 \pm 0.24 \mathrm{~mA}$, resistance of $521 \pm 77$ ohms and $\mathrm{R}$ wave of $10.2 \pm 3.9 \mathrm{mV}$.

Similar to our results, Perrins et $\mathrm{a}^{6}$ noted that the ventricular screw-in lead (Medtronic 6957) displayed a higher acute pacing threshold than the tined lead (Medtronic 6961/2, p <0.001), which they attributed to its larger surface area. Using the same screw-in lead, Bisping et $\mathrm{al}^{7}$ found no significant difference between the pacing threshold in the atrium and ventricle. A number of investigators including Santini, ${ }^{8}$ Bisping, ${ }^{7}$ Kleinert ${ }^{9}$ and their co-workers have noted that there is a significant decrease in the atrial pacing threshold and increase in the amplitude of the $P$ wave over a period of 10 to 30 minutes after implantation of a screw-in lead in the atrium. These changes were noted to coincide with the disappearance of the injury potential. ${ }^{7}$ However, although these features have been recognized in the past, our study is the first to provide a systematic study of these phenomena.

Shandling et al ${ }^{10}$ compared the unipolar P-wave amplitude at the time of atrial lead placement in 43 patients to that recorded at the end of pacemaker surgery. Overall, there was an increase in the mean P-wave amplitude in patients with passive fixation leads but there was considerable individual fluctuation. They ${ }^{10}$ recommended that the atrial sensitivity be programmed with an adequate margin of safety early after pacemaker implantation.

Limitations: The leads of only 1 manufacturer were examined, and therefore our findings may not apply to other types of leads. Another limitation is that we did not examine the pacing or sensing characteristics of a bipolar screw-in lead. However, available data, including those in the present study, suggest that there is little difference in pacing threshold and sensing characteristics between the unipolar and bipolar varieties of a particular type of lead."

Acknowledgment: We are grateful to Beverly Burgie and the staff of the electrophysiology laboratory for technical assistance and to Suzanna Susock and Vanessa Sims for secretarial assistance.

\section{REFERENCES}

1. Milliken GA, Johnson DE. Analysis of messy data. Volume I: Designed Experiments. New York: Van Nostrand Reinold, 1986:322-350.

2. Mond H, Sloman $\mathrm{G}$. The small-tined pacemaker lead-absence of dislodgement. PACE 1980;3:171-177.

3. Berman ND, Dickson SE, Lipton IH. Acute and chronic clinical performance comparison of a porous and a solid electrode design. PACE 1982,5.67-71.

4. Scoblionko DP, Rolett EL. Short-term threshold behavior of human ventricular pacing electrodes: noninvasive monitoring with a multiprogrammable pacing system. PACE 1981:4:631-637.

5. Holmes DR Jr, Nissen RG, Maloney JD, Broadbent JC, Merideth J. Transvenous tined electrode systems. An approach to acute dislodgement. Mayo Clin Proc 1979;54:219-222.

6. Perrins EJ, Sutton R, Kalebic B, Richards LR, Morley C, Terpstra B. Modern atrial and ventricular leads for permanent cardiac pacing. $\mathrm{Br}$ Heart $J$ 1981:46:196-201.

7. Bisping HJ, Kreuzer J, Birkenheier H. Three-year clinical experience with a new endocardial screw-in lead with introduction protection for use in the atrium and ventricle. $P A C E$ 1980;3:424-435.

8. Santini M, Rocchi M, Alliegro A, Adinolfi E, Pandolfo L, Masini V. Reliability of screw-in leads for permanent atrial sensing and pacing. In: Feruglio GA, ed. Cardiac Pacing: Electrophysiology and Pacemaker Technology. Padova, Italy: Piccin Medical Books, 1982:631-635.

9. Kleinert M. Permanent atrial leads. PACE 1980;3:487-491.

10. Shandling AH, Castellanet MJ, Thomas LA, Mulvihill DF, Feuer JM, Messenger JC. Variation in $\mathrm{P}$ wave amplitude immediately after pacemaker implantation: possible mechanism and implications for early programming. PACE 1989;12:1797-1805

11. Ohm OJ. Pacemaker technology: pacing leads. Cardiovasc Clin 1983; $14: 247-264$ 